UNITU-THEP-17/1998

\title{
Magnetic Monopoles and Topology of Yang-Mills Theory in Polyakov Gauge
}

\author{
M. Quandt用, H. Reinhardt, A. Schäfke* \\ Institut für Theoretische Physik, Universität Tübingen \\ D-72076 Tübingen, Germany.
}

\begin{abstract}
We express the Pontryagin index in Polyakov gauge completely in terms of magnetically charged gauge fixing defects, namely magnetic monopoles, lines, and domain walls. Open lines and domain walls are topologically equivalent to monopoles, which are the genuine defects. The emergence of non-genuine magnetically charged closed domain walls can be avoided by choosing the temporal gauge field smoothly. The Pontryagin index is then exclusively determined by the magnetic monopoles.
\end{abstract}

Keywords: Yang-Mills theory, magnetic monopoles, Pontryagin index

*Supported by "Graduiertenkolleg: Hadronen und Kerne" 


\section{Introduction}

Recent lattice calculations [1] give evidence that confinement is realized as a dual Meissner effect, at least in the so-called Abelian gauges [2]. In these gauges magnetic monopoles arise as obstructions to fixing the coset $G / H$ of the gauge group $G$, where $H$ is the Cartan subgroup which is left invariant. Lattice calculations indicate that these monopoles are in fact condensed [3], a necessary condition for the QCD vacuum forming a dual superconductor.

Magnetic monopoles are long ranged fields and should hence contribute to the topological properties of gauge fields. Furthermore topologically non-trivial field configurations can explain spontaneous breaking of chiral symmetry 谏. It is therefore interesting to clarify to which extent magnetic monopoles contribute to the topology of gauge fields.

Previously it was shown by one of us that in the Polyakov gauge,

$$
\Omega(\mathbf{x})=\mathrm{P} \exp \left(-\int d x_{0} A_{0}\right) \stackrel{!}{=} \operatorname{diag},
$$

which is a particular Abelian gauge, magnetic monopoles completely account for the non-trivial topology of Yang-Mills fields. To be specific, consider a pure Yang-Mills theory with colour group $S U(2)$. In Polyakov gauge the magnetic monopoles arise at those points in three-space where the Polyakov loop $\Omega(\mathbf{x})$ becomes an irregular element of the gauge group, $\Omega\left(\mathbf{x}_{i}\right)=(-\mathbb{1})^{n_{i}}= \pm \mathbb{1}$, i.e. a centre element for $S U(2)$. Here $n_{i}$ is an integer. Furthermore, in order for the Pontryagin index to be well defined and the action finite, the gauge fields have to become asymptotically a pure gauge, which in turn implies that the Polyakov loop approaches an angle independent value at spatial infinity,

$$
\lim _{r \rightarrow \infty} \Omega(r, \hat{\mathbf{x}})=(-\mathbb{1})^{n_{0}} .
$$

The following expression was derived for the Pontryagin index [5]

$$
\nu=-\sum_{i} \ell_{i} m_{i}
$$

where $m_{i}$ denotes the magnetic charge of the monopole and $\ell_{i}=n_{i}-n_{0}$ is an integer which can be interpreted as the invariant length traced out by the Dirac string in group space.

The boundary condition (2) allows us to compactify our spatial manifold

$$
\mathbb{R}^{3} \rightarrow \dot{\mathbb{R}}^{3}=\mathbb{R}^{3} \cup\{\infty\} \simeq S^{3} .
$$

In this way, the surface at spatial infinity becomes a point of the compactified manifold, $\dot{\mathbb{R}}^{3} \simeq S^{3}$, which hosts a magnetic monopole due to the b.c. (2). Furthermore on a compact manifold the net magnetic charge of all monopoles has to vanish, $\sum_{i} m_{i}=0$. Given this setting, the expression for the Pontryagin index found in ref. [5] simplifies to

$$
\nu=-\sum_{i} m_{i} n_{i}
$$

where the summation is now over all magnetic monopoles including the one at the infinitely distant point.

Subsequently the same problem has been treated in a somewhat different fashion in references [6, 7] resulting in the following expression for the Pontryagin index,

$$
\nu=-\sum_{\substack{i \\ \Omega=-\mathbb{1}}} m_{i},
$$


where the summation is performed over the charges $m_{i}$ of magnetic monopoles corresponding to the irregular element $\Omega=-\mathbb{1}$ while in eq. (5) the summation is over all monopoles. In addition the invariant length of the Dirac string enters only in eq. (5). Formally eq. (6) results from eq. (司) by restricting the integers $n_{k}$ to $n_{k}=0,1$.

In the present paper we will summarize the result of a thorough investigation of the topological charge in the presence of gauge fixing defects. In particular we will show that eq. (5) is more general than eq. (6). While the latter formula gives the correct winding number only in the absence of domain walls, eq. (5) includes already the effect of non-genuine domain walls, which arise when $\ln \Omega$ is restricted to first Weyl alcove.

\section{Abelian Gauge Fixing}

The starting point of the (canonical) quantization of Yang-Mills theory is the Weyl-gauge [8]

$$
A_{0}=0
$$

It is generally assumed that in this gauge the dynamical fields, i.e. the spatial field components $A_{i=1,2,3}(x)$ are smooth functions of space-time. The quantity of interest is the gauge invariant partition function for which it is straightforward to derive the following functional integral representation [9, 10]:

$$
Z=\int_{G} \mathcal{D} \mu[\Omega] \cdot e^{-i n[\Omega] \theta} \underset{\text { b.c. }(\Omega)}{ } \mathcal{D} \mathbf{A} \exp \left(-S_{\mathrm{YM}}\left[A_{0}=0, \mathbf{A}\right]\right) .
$$

Gauge invariance requires here the spatial gauge fields to satisfy the twisted boundary condition

$$
\mathbf{A}(t=0, \mathbf{x})=(\mathbf{A}(t=\beta, \mathbf{x}))^{\Omega},
$$

where $A^{\Omega}=\Omega A \Omega^{\dagger}+\Omega \partial \Omega^{\dagger}$ is the gauge transformed field, and further requires to integrate over all gauge functions $\Omega(\mathbf{x})$ with the invariant (Haar) measure $\mu$. Like the dynamical fields $A_{i=1,2,3}(x)$, the gauge rotation $\Omega(\mathbf{x})$ can be assumed to be smooth.

Topologically the gauge fields are classified by the Pontryagin index

$$
\nu[A]=-\frac{1}{8 \pi^{2}} \int_{\mathcal{M}} \operatorname{tr}(F \wedge F)=\frac{1}{32 \pi^{2}} \int_{\mathcal{M}} d^{4} x F_{\mu \nu}^{a *} F_{\mu \nu}^{a},
$$

where $\mathcal{M}$ is the space-time manifold which we choose to be $\mathcal{M}=[0, \beta] \times \mathrm{M}$ with the spatial threemanifold $M$. We shall specify $M$ as the one-point compactification $M=\mathbb{R}^{3} \cup\{\infty\}=\dot{\mathbb{R}}^{3} \simeq S^{3}$.

In the Weyl gauge and with the twisted boundary condition (9) the Pontryagin index is given by the winding number

$$
n[\Omega]=-\frac{1}{24 \pi^{2}} \int_{\mathrm{M}} \operatorname{tr}(L \wedge L \wedge L) ; \quad L=\Omega \cdot d \Omega^{-1}
$$

\footnotetext{
${ }^{1} \mathrm{~A}$ Weyl alcove is a fundamental domain in the Cartan algebra with respect to the extended Weyl group, i.e. any of the discrete symmetries (displacements or Weyl symmetries) leads out of the alcove. For $G=S U(2)$ as an example, the Cartan group is $\left\{e^{i \chi \sigma_{3}}\right\}$, the displacements are $\chi \rightarrow \chi+2 \pi m$ and the Weyl symmetry is $\chi \rightarrow-\chi$ whence the Weyl alcove is found to be $\chi \in[0, \pi]$.
} 
of the gauge function $\Omega(\mathbf{x})$, i.e.

$$
\nu\left[A_{0}=0, \mathbf{A}\right]=n[\Omega] .
$$

Note that this number enters with the vacuum angle $\theta$ in the partition function (8).

For many purposes the twisted boundary conditions are inconvenient and it is useful to convert them to periodic ones,

$$
\mathbf{A}(t=\beta, \mathbf{x})=\mathbf{A}(t=0, \mathbf{x}),
$$

by performing the following time-dependent gauge transformation [9, 10]

$$
U(t, \mathbf{x})=\Omega(\mathbf{x})^{\frac{t}{\beta}-1}
$$

which introduces a time-independent temporal gauge field component

$$
A_{0}^{\prime}=\left(A_{0}=0\right)^{U}=U \partial_{0} U^{-1}=-\beta^{-1} \ln \Omega(\mathbf{x}) .
$$

From the gauge defined by the previous equation, which is equivalent to

$$
\partial_{0} A_{0}=0
$$

one arrives at the Polyakov gauge by a time-independent gauge transformation $V(\mathbf{x})$ diagonalizing $\Omega(\mathbf{x})$ and hence $A_{0}$,

$$
\Omega(\mathbf{x})=V^{-1}(\mathbf{x}) \cdot \omega(\mathbf{x}) \cdot V(\mathbf{x}) \stackrel{V}{\longrightarrow} \omega(\mathbf{x})
$$

where $\omega$ and $V$ live in the Cartan subgroup $H \subset G$ and in the coset $G / H$, respectively. The coset element $V \in G / H$ which diagonalizes $\Omega \in G$ is obviously defined only up to an element of the normalizer $N(H)$ of $H$ in $G$,

$$
V \rightarrow g \cdot V, \quad g \in N(H) .
$$

The normalizer $N(H)$ is related to the Cartan subgroup $H$ by $N(H)=W \times H$ where $W$ denotes the Weyl group. For the gauge group $G=S U(N)$ the Weyl group $W$ is isomorphic to the permutation group $S_{N}$. In fact, the Weyl transformations $w \in W$ permute the diagonal elements of $\omega$ and are not part of the Cartan subgroup.

Topological obstructions to implementing the Polyakov gauge occur, and these are of three different types:

1. The gauge function $\Omega(\mathbf{x})$ may take values corresponding to irregular elements of the gauge group in which two eigenvalues coincide and the diagonalization, i.e. the coset element $V \in G / H$, is not well defined.2 In this case we have local gauge fixing defects, which manifest themselves as magnetic charges in the induced gauge field $\mathcal{A}_{i}=V \partial_{i} V^{\dagger}$.

2. In the diagonalization $\Omega=V^{-1} \omega V$ the elements $\omega$ and $V$ may not be globally defined and smooth on $\mathrm{M}$ even if $\Omega(\mathbf{x})$ is smooth and everywhere regular. The point is that the compactification imposes certain boundary conditions on $\Omega(\mathbf{x})$, and $\omega$ or $V$ may fail to obey these conditions.

\footnotetext{
${ }^{2}$ For the gauge group $G=S U(2)$ the irregular elements coincide with the centre elements $\pm \mathbb{1}$.
} 
3. Functions of matrices like $\Omega(\mathbf{x})$ are generally defined by the spectral theorem, i.e. even if diagonalization problems on $\mathrm{M}$ are absent, $f(\Omega)$ can only be smooth if the function $f$ is holomorphic on the spectrum of $\Omega(\mathbf{x})$. For the fractional power in eq. (14) or the logarithm in eq. (15) this may be impossible due to the branch cut of the logarithm in the complex plane.

The second type of obstructions has been discussed in ref. [11] and we will not consider it here. Furthermore we will see below that the third type of obstruction is automatically resolved by a proper treatment of the defects arising in item one, and hence we will concentrate in the following on the investigation of the local gauge fixing defects. We will in particular show that in the Polyakov gauge the Pontryagin index arises entirely from these gauge defects.

Note that the gauge potentials in both the gauge (16) and the Polyakov gauge (17) fulfill the periodic boundary conditions (13) and thus live on the closed compact manifold $\mathcal{M}=S^{1} \times \mathrm{M}$. It is then easy to see that the Pontryagin index arises exclusively from the defects. As is well known, the integrand in the Pontryagin index is a total derivative for non-singular gauge fields,

$$
\operatorname{tr}(F \wedge F)=d \mathrm{~K}[A]
$$

where

$$
\mathrm{K}=\operatorname{tr}\left(F \wedge A-\frac{1}{3} A \wedge A \wedge A\right)
$$

is the topological current. From $\partial \mathcal{M}=0$ and Stokes' theorem we find

$$
\nu=-\frac{1}{8 \pi^{2}} \int_{\mathcal{M}} d \mathrm{~K}=-\frac{1}{8 \pi^{2}} \int_{\partial \mathcal{M}} \mathrm{K}=0 .
$$

The crucial observation is here that the gauge fixing defects for which the coset element $V \in G / H$ is ill-defined give rise to singular connections $A^{V}$ in Polyakov gauge (cf. eq. (17)), so that at the gauge defects equation (19) does not apply.

A similar conclusion may be drawn from the results of ref. [5] where it was shown that the Pontryagin index, which is trivially invariant under small gauge rotations, also does not change under both transformations (14) and (17), i.e.

$$
\nu\left[A^{U}\right]=\nu\left[\left(A^{U}\right)^{V}\right]=\nu[A] \stackrel{(12)}{=} n[\Omega] .
$$

In the Polyakov gauge, however, the gauge function $\Omega(\mathbf{x})$ is diagonalized and from (17) we infer

$$
n[\Omega]=n\left[V^{-1}\right]+n[\omega]+n[V]=n[\omega]=0
$$

unless the coset transformation $V(\mathbf{x})$ is singular somewhere on the spatial manifold M. Again we conclude that the winding number of $\Omega$ and thus the Pontryagin index in Polyakov gauge arises exclusively from the defects.

\section{$3 \quad$ Gauge Fixing Defects}

A group element is called irregular when two of its eigenvalues are degenerate. For irregular $\Omega(\mathbf{x})$ we can always consider the two degenerate eigenvalues of $\Omega(\mathbf{x})$ to belong to an $S U(2)$ subgroup of the full gauge group $G=S U(N)$. Therefore it suffices to consider the gauge group 


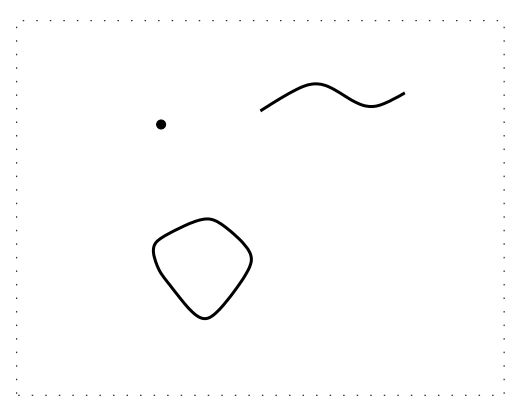

(a)

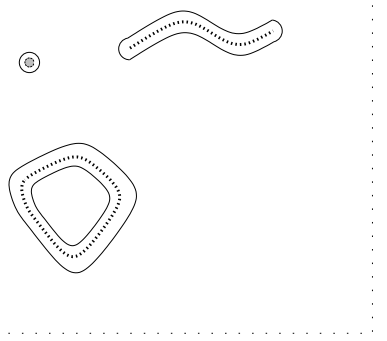

(b)

Figure 1: Wrapping of some generic diagonalisation defects. (a) Point defects and open or closed line defects, (b) wrapping of these defects by closed surfaces with infinitesimal volume $\epsilon$.

$S U(2)$ where the irregular elements are given by the centre elements $\Omega= \pm \mathbb{1}$. We define an individual defect $\mathrm{D}_{i}$, as usual, as a connected set of points for which the smooth mapping $\Omega(\mathbf{x})$ takes on an irregular element,

$$
\mathrm{D}_{i}=\{\mathrm{x} \in \mathrm{M}, \Omega(\mathbf{x})=\text { const }= \pm \mathbb{1}\} \subseteq \mathrm{M}, \quad \mathrm{D}_{i} \text { connected. }
$$

Since $\Omega(\mathbf{x})$ is time-independent, all defects are static and it suffices to investigate the threedimensional space $\mathrm{M}$. According to the dimensionality we distinguish the following defects:

- $\pi_{2}\left(\mathrm{M} \backslash \mathrm{D}_{i}\right) \neq \emptyset$ : Isolated point defects (magnetic monopoles)

- $\pi_{1}\left(\mathrm{M} \backslash \mathrm{D}_{i}\right) \neq \emptyset$ : Closed line defects

- $\pi_{0}\left(\mathrm{M} \backslash \mathrm{D}_{i}\right) \neq \emptyset$ : Closed domain walls

Open line and wall defects are topologically equivalent to isolated point defects. Similarly, three-dimensional defects give merely rise to additional internal boundaries of $\mathrm{M}$ where the gauge function $\Omega(\mathbf{x})$ takes an irregular element. The volume of the three-dimensional defects does not contribute to the winding number, since $\Omega(\mathbf{x})$ is a constant $\pm \mathbb{1}$ there. Such volume defects can therefore be treated analogously to the point defects and will not be considered here.

To proceed further we wrap the defects by closed surfaces which are infinitesimally close to the defects, see fig. 1. The defect $D_{i}$ together with its wrapping is denoted by $D_{i}^{\epsilon}$. We then cut out the defects together with their wrappings from our spatial manifold $M$ giving rise to the punctured space

$$
\overline{\mathrm{M}}_{\epsilon}=\mathrm{M} \backslash \bigcup_{i} \mathrm{D}_{i}^{\epsilon}
$$

where each defect gives rise to an internal surface enclosing the defect, see fig. 1. We will assume that the punctured space $\bar{M}_{\epsilon}$ admits a covering $\left\{\mathrm{X}_{\alpha}\right\}$ by closed contractible sets $\mathrm{X}_{\alpha}$

$$
\overline{\mathrm{M}}_{\epsilon}=\bigcup_{\alpha} \mathrm{X}_{\alpha}
$$

with

$$
\mathbf{X}_{\alpha} \cap \mathbf{X}_{\beta}=\partial \mathbf{X}_{\alpha} \cap \partial \mathbf{X}_{\beta}
$$

such that on each (topologically trivial) patch $\mathrm{X}_{\alpha}$ we have smooth diagonalization maps $V_{\alpha}(\mathbf{x})$ and $\omega_{\alpha}(\mathbf{x})$ with $\Omega=V_{\alpha}^{-1} \omega_{\alpha} V_{\alpha}$. The triangulation (25) implies that, according to (26), the 
closed oriented patches $\mathrm{X}_{\alpha}$ intersect precisely on their boundaries. Note that the patches $\mathrm{X}_{\alpha}$ are oriented, and so is their intersection. Contrary to ordinary sets, the intersection operator does therefore not commute,

$$
\mathrm{X}_{\alpha} \cap \mathrm{X}_{\beta}=-\mathrm{X}_{\beta} \cap \mathrm{X}_{\alpha} .
$$

In the presence of closed domain walls the manifold $\bar{M}_{\epsilon}$ consists of disconnected pieces $M_{a}$, $\mathrm{M}=\bigcup_{a} \mathrm{M}_{a}$ separated by the domain walls. In each connected component $\mathrm{M}_{a}$ the induced gauge field $a_{0}=-\frac{1}{\beta} \ln \omega(\mathbf{x})$ can be chosen smoothly, but some care has to be taken when extending $\omega(\mathbf{x})$ and $\ln \omega(\mathbf{x})$ over different domains $\mathrm{M}_{a}$.

To see this, recall that $\Omega(\mathbf{x})$ is smooth over the common boundary of two patches $\mathrm{X}_{\alpha}$ and $\mathrm{X}_{\beta}$, and we have

$$
\Omega(\mathbf{x})=V_{\alpha}^{-1}(\mathbf{x}) \omega_{\alpha}(\mathbf{x}) V_{\alpha}(\mathbf{x})=V_{\beta}^{-1}(\mathbf{x}) \omega_{\beta}(\mathbf{x}) V_{\beta}(\mathbf{x})
$$

so that the diagonal maps $\omega_{\alpha}(\mathbf{x})$ are related by

$$
\omega_{\alpha}(\mathbf{x})=h_{\alpha \beta}(\mathbf{x}) \omega_{\beta}(\mathbf{x}) h_{\alpha \beta}^{-1}(\mathbf{x})
$$

with the transition functions

$$
h_{\alpha \beta}(\mathbf{x})=V_{\alpha}(\mathbf{x}) \cdot V_{\beta}^{-1}(\mathbf{x}) .
$$

They obviously satisfy the co-cycle condition

$$
h_{\alpha \beta} \cdot h_{\beta \gamma}=h_{\alpha \gamma} .
$$

From eq. (29) we infer that $h_{\alpha \beta}$ takes values in the normalizer $N=W \times H$ of the Cartan subgroup $H$ and consequently, the diagonalizations $\omega_{\alpha}$ and $\omega_{\beta}$ coincide up to a Weyl transformation. Since our color group is simply connected, $\pi_{1}(S U(N))=\emptyset$, the picture $P \subset H$ of a Weyl alcove under the exponential map represents a fundamental domain for the Cartan group, i.e. any Weyl transformation leads out of $P$. Thus, by restricting our diagonalization $\omega$ to $P \subset H$, it is possible to choose

$$
\omega_{\alpha}=\omega_{\beta}
$$

smoothly on the overlap of two patches. Furthermore, since the branch cut of the logarithm is situated at the defects, which are excluded from our manifold $\overline{\mathrm{M}}_{\epsilon}$, and since the subset $P \subset H$ is simply connected, the same is true for the logarithm of our diagonalization,

$$
\ln \omega_{\alpha}=\ln \omega_{\beta} .
$$

On the other hand, there are no overlapping patches between different domains $\mathrm{M}_{a}$ (disconnected by closed domain walls), and eqs. (32) and (33) do no longer hold necessarily. In fact, there is an ambiguity in the choice of fundamental subsets $P \subset H$ for the diagonalization in the various connected regions $\mathrm{M}_{a}$. As a consequence, the maps $\omega_{a}$ and $\omega_{b}$ at infinitesimally close points on opposite sides of a closed domain wall are related by

$$
\omega_{a}=\omega_{b} \quad \text { or } \quad \omega_{a}=\omega_{b}^{\dagger}
$$

and

$$
\ln \omega_{a}= \pm \ln \omega_{b}+2 \pi i k \sigma_{3}, \quad k \in \mathbb{Z}
$$




\section{The Winding Number Expressed by Defects}

The integrand in the winding number $n[\Omega]$ can be locally (i.e. within a patch $\mathrm{X}_{\alpha}$ ) expressed as a total derivative

$$
\operatorname{tr}(L \wedge L \wedge L)=d \mathrm{G}\left(\omega, V_{\alpha}\right)
$$

where

$$
\mathrm{G}\left[\omega, V_{\alpha}\right]=-6 \operatorname{tr}\left(\mathcal{A}_{\alpha} \wedge \omega d \omega^{-1}\right)+3 \operatorname{tr}\left(\mathcal{A}_{\alpha} \omega^{-1} \wedge \mathcal{A}_{\alpha} \omega\right), \quad \mathcal{A}_{\alpha}=V_{\alpha} d V_{\alpha}^{-1} .
$$

Applying Stokes' theorem, we find

$$
n[\Omega]=-\frac{1}{24 \pi^{2}} \sum_{\alpha} \int_{\partial \mathbf{X}_{\alpha}} \mathrm{G}\left[\omega, V_{\alpha}\right] .
$$

A patch $\mathrm{X}_{\alpha}$ can have a common border with another patch $\mathrm{X}_{\beta}$ or with a defect $\mathrm{D}_{i}^{\epsilon}$ whence we find for the surface of a patch ${ }^{3}$

$$
\partial \mathrm{X}_{\alpha}=\sum_{\beta \neq \alpha} \mathrm{X}_{\alpha} \cap \mathrm{X}_{\beta}+\sum_{i} \mathrm{X}_{\alpha} \cap \mathrm{D}_{i}^{\epsilon}
$$

Using this decomposition of the surface $\partial \mathrm{X}_{\alpha}$ we obtain

$$
n[\Omega]=-\frac{1}{48 \pi^{2}} \sum_{\alpha, \beta} \int_{\mathrm{X}_{\alpha} \cap \mathrm{X}_{\beta}}\left(\mathrm{G}\left[\omega, V_{\alpha}\right]-\mathrm{G}\left[\omega, V_{\beta}\right]\right)-\frac{1}{24 \pi^{2}} \sum_{\alpha, i} \int_{\mathrm{X}_{\alpha} \cap \mathrm{D}_{i}^{\epsilon}} \mathrm{G}\left[\omega, V_{\alpha}\right],
$$

where we exploited the different orientation (27) of the intersection $\mathrm{X}_{\alpha} \cap \mathrm{X}_{\beta}$, as seen from $\mathrm{X}_{\alpha}$ and $\mathrm{X}_{\beta}$, respectively. From (35) and (30) we find

$$
\mathrm{G}\left[\omega, V_{\alpha}\right]-\mathrm{G}\left[\omega, V_{\beta}\right]=-6 d \operatorname{tr}\left(h_{\alpha \beta} d h_{\alpha \beta}^{\dagger} \ln \omega\right) .
$$

Using the cocycle condition (31), the first integral in (38) can be rewritten by means of Stokes' theorem,

$$
\int_{\mathrm{X}_{\alpha} \cap \mathrm{X}_{\beta}}\left(\mathrm{G}\left[\omega, V_{\alpha}\right]-\mathrm{G}\left[\omega, V_{\beta}\right]\right)=-6 \sum_{i} \int_{\mathrm{X}_{\alpha} \cap \mathrm{X}_{\beta} \cap \mathrm{D}_{i}^{\epsilon}} \operatorname{tr}\left(h_{\alpha \beta} d h_{\alpha \beta}^{\dagger} \ln \omega\right) .
$$

Here we have decomposed the boundary of $\mathrm{X}_{\alpha} \cap \mathrm{X}_{\beta}$ as (cf. eq. (37))

$$
\partial\left(\mathrm{X}_{\alpha} \cap \mathrm{X}_{\beta}\right)=\bigcup_{\gamma} \mathrm{X}_{\alpha} \cap \mathrm{X}_{\beta} \cap \mathrm{X}_{\gamma}+\bigcup_{i} \mathrm{X}_{\alpha} \cap \mathrm{X}_{\beta} \cap \mathrm{D}_{i}^{\epsilon}
$$

Turning to the second integral in (38) we observe that the last term in eq. (35) does not contribute, since it vanishes for $\epsilon \rightarrow 0$, i.e. $\omega \rightarrow \pm \mathbb{1}$. On the other hand the first term in (35) can be written as

$$
-6 \operatorname{tr}\left(\mathcal{A}_{\alpha} \wedge \omega d \omega^{-1}\right)=-6 d \operatorname{tr}\left(\mathcal{A}_{\alpha} \ln \omega\right)+6 \operatorname{tr}\left(d \mathcal{A}_{\alpha} \ln \omega\right) .
$$

Hence by using Stokes' theorem, we obtain for the second integral in (38)

$$
\sum_{\alpha, i} \int_{\mathrm{X}_{\alpha} \cap \mathrm{D}_{i}^{\epsilon}} \mathrm{G}\left[\omega, V_{\alpha}\right]=-6 \sum_{\alpha, i} \int_{\partial\left(\mathrm{X}_{\alpha} \cap \mathrm{D}_{i}^{\epsilon}\right)} \operatorname{tr}\left(\mathcal{A}_{\alpha} \ln \omega\right)+6 \sum_{\alpha, i} \int_{\mathrm{X}_{\alpha} \cap \mathrm{D}_{i}^{\epsilon}} \operatorname{tr}\left(d \mathcal{A}_{\alpha} \ln \omega\right) .
$$

\footnotetext{
${ }^{3}$ Note that this equation defines an orientation for the intersection operator of the (oriented) patches.
} 
Expressing the surface $\partial\left(\mathrm{X}_{\alpha} \cap \mathrm{D}_{i}^{\epsilon}\right)$ analoguously to eq. (41) and taking care of the proper orientation of the intersections (cf. (27)), we have

$$
\partial\left(\mathrm{X}_{\alpha} \cap \mathrm{D}_{i}^{\epsilon}\right)=-\sum_{\beta} \mathrm{X}_{\alpha} \cap \mathrm{X}_{\beta} \cap \mathrm{D}_{i}^{\epsilon}
$$

and the first term on the r.h.s. of (43) becomest

$$
-6 \sum_{\alpha, i} \int_{\partial\left(\mathrm{X}_{\alpha} \cap \mathrm{D}_{i}^{\epsilon}\right)} \operatorname{tr}\left(\mathcal{A}_{\alpha} \ln \omega\right)=+3 \sum_{\alpha, \beta,{ }_{\mathrm{X}_{\alpha} \cap \mathrm{X}_{\beta} \cap \mathrm{D}_{i}^{\epsilon}}} \operatorname{tr}\left(\left(\mathcal{A}_{\alpha}-\mathcal{A}_{\beta}\right) \ln \omega\right) .
$$

Using eq. (30) this term is seen to cancel eq. (40) so that the winding number (38) receives a non-vanishing contribution only from the second term in (43),

$$
n[\Omega]=-\frac{1}{4 \pi^{2}} \sum_{\alpha, i} \int_{\mathbf{X}_{\alpha} \cap D_{i}^{\epsilon}} \operatorname{tr}\left(\ln \omega \cdot T^{3}\right) \cdot d \mathcal{A}_{\alpha}^{3}, \quad \mathcal{A}_{\alpha}=V_{\alpha} \cdot d V_{\alpha}^{-1} .
$$

On a defect $\mathrm{D}_{i}, \Omega(\mathbf{x})$ and hence $\omega(\mathbf{x})$ are irregular i.e. $\omega(\mathbf{x})$ takes values $(-\mathbb{1})^{n_{i}}= \pm \mathbb{1}$ (for $G=S U(2))$ and is constant. Hence we obtain

$$
\left.\operatorname{tr}\left(\ln \omega \cdot T^{3}\right)\right|_{\mathrm{D}_{i}^{\epsilon}}=\pi n_{i}
$$

Furthermore the quantity

$$
m_{i}=\frac{1}{4 \pi} \sum_{\alpha} \int_{\mathrm{X}_{\alpha} \cap \mathrm{D}_{i}^{\epsilon}} d \mathcal{A}_{\alpha}^{3}
$$

is the magnetic flux through the wrapping surface of the defect and hence represents the magnetic charge of the defect. Applying again Stokes' theorem and eq. (44) the flux can be expressed as

$$
m_{i}=-\frac{1}{8 \pi} \sum_{\alpha, \beta} \int_{\mathbf{X}_{\alpha} \cap \mathbf{X}_{\beta} \cap \mathrm{D}_{i}^{\epsilon}}\left(\mathcal{A}^{3}\left[V_{\alpha}\right]-\mathcal{A}^{3}\left[V_{\beta}\right]\right)=\frac{1}{4 \pi} \sum_{\alpha, \beta} \int_{\mathbf{X}_{\alpha} \cap \mathbf{X}_{\beta} \cap \mathrm{D}_{i}^{\epsilon}} \operatorname{tr}\left(h_{\alpha \beta} d h_{\alpha \beta}^{-1} \cdot T^{3}\right) .
$$

Inserting eq. (47) and (48) into equation (46) we obtain for the winding number

$$
n[\Omega]=-\sum_{i} n_{i} m_{i}
$$

This is precisely the result derived in ref. [5] for a compact spatial manifold M. Let us stress that, as the above derivation reveals, all defects with $n_{i} \neq 0$ carrying non-zero magnetic charge $m_{i} \neq 0$ contribute to the topological charge.

Since a shift of $\ln \omega$ by $2 \pi i$ leads to the same $\omega(\mathbf{x})$, we have a freedom in the choice of $\chi=-i \ln \omega$. Choosing $\chi$ smooth at the defect where $\omega= \pm \mathbb{1}$ will in general lead $\chi$ outside

\footnotetext{
${ }^{4}$ The relative sign in the last integral is again due to the opposite orientation of the common boundary, as seen from the adjacent patches.

${ }^{5}$ Note that the normal vector on the wrapping surface around the defect $D_{i}^{\epsilon}$ points out of the punctured space $\overline{\mathrm{M}}_{\epsilon}$, i.e. towards the defect. By contrast, the intersection $\mathrm{X}_{\alpha} \cap \mathrm{D}_{i}^{\epsilon}$ is oriented opposite to $\partial \mathrm{D}_{i}^{\epsilon}$ (cf. eq. (37)), so that our definition (48) yields the usual sign of the magnetic charge, i.e. the magnetic flux emanating from the defect.
} 
the first Weyl alcove. As we will illustrate below in this case closed domain walls will carry no magnetic charge and hence do not contribute to the winding number.

Alternatively we can restrict $\chi$ to the first Weyl alcove $\chi \in[0, \pi]$. Then at the defect the integer defined in equation (47) is restricted to $n_{i}=0,1$ and equation (50) becomes

$$
n[\Omega]=-\sum_{\substack{i \\ \Omega=-\mathbb{1}}} m_{i},
$$

i.e. only the defects with $\Omega=-\mathbb{1}$ contribute. Restricting $\chi$ to the first Weyl alcove $\chi \in[0, \pi]$ implies that $\chi$ is continuous but not necessarily smooth at the defect, see fig. 3. In this case closed domain walls with $\omega=-\mathbb{1}$ now carry twice the magnetic charge of a monopole and hence contribute to $n[\Omega]$ as will be illustrated below.

Thus in the generic case where only magnetic monopoles and domain walls are present, eq. (51) can be more explicitly written as

$$
n[\Omega]=-\sum_{\substack{k \text { (magnetic monopoles }) \\ \Omega=-\mathbb{1}}} m_{k}-\sum_{\substack{k(\text { domain walls }) \\ \Omega=-\mathbb{1}}} m_{k} .
$$

We observe that for the smooth parameterization the magnetic monopoles fully account for the topological charge (50), while there is an extra contribution in eq. (52) due to magnetically charged domain walls. In refs. [6, 7] this domain wall contribution to $n[\Omega]$ was not included although $\chi=-i \ln \omega$ was restricted to first Weyl alcove.

\section{$5 \quad$ Hedgehog Field as Generic Example}

Let us finally illustrate our result for the two different methods eqs. (50) and (51) by means of a specific example. The prototype of smooth maps $\Omega: \dot{\mathbb{R}}^{3} \rightarrow S U(2)$ with non-vanishing winding number is provided by the well-known hedgehog configuration,

$$
\Omega(\mathbf{x})=\exp (i \chi(r) \cdot \hat{\mathbf{x}} \boldsymbol{\sigma})=\mathbb{1} \cdot \cos \chi(r)+i \hat{\mathbf{x}} \boldsymbol{\sigma} \cdot \sin \chi(r) ; \quad r \equiv|\mathbf{x}| .
$$

For this map to be smooth, we have to avoid the singularity at the origin where $\hat{\mathbf{x}}$ is ill-defined,

$$
\sin \chi(0)=0 \quad \Longleftrightarrow \quad \chi(0)=n_{0} \cdot \pi ; \quad n_{0} \in \mathbb{Z} .
$$

Furthermore, the compactification $\mathbb{R}^{3} \rightarrow \dot{\mathbb{R}}^{3}$ implies that $\Omega(\mathbf{x})$ be angle-independent as $r=$ $|\mathbf{x}| \rightarrow \infty$, i.e. the $\hat{\mathbf{x}}$-dependent part of $\Omega$ must vanish as $r \rightarrow \infty$, whence

$$
\sin \chi(\infty)=0 \quad \Longleftrightarrow \quad \chi(\infty)=n_{\infty} \cdot \pi ; \quad n_{\infty} \in \mathbb{Z} .
$$

The winding number of such a hedgehog map is determined by the difference of the two integers from the profile boundary conditions via $n[\Omega]=n_{\infty}-n_{0}$. For definiteness we choose

$$
n_{0}=0, \quad \text { i.e. } \quad n_{\infty}=n[\Omega] .
$$

As expected, there is a continuous diagonalisation of this map,

$$
\omega(\mathbf{x})=\omega(r)=\exp \left(i \tilde{\chi}(r) \sigma_{3}\right)
$$

where the profile $\tilde{\chi}$ reflects our choice of Weyl alcoves in the connected regions separated by the domain walls. There are two basically different choices: 

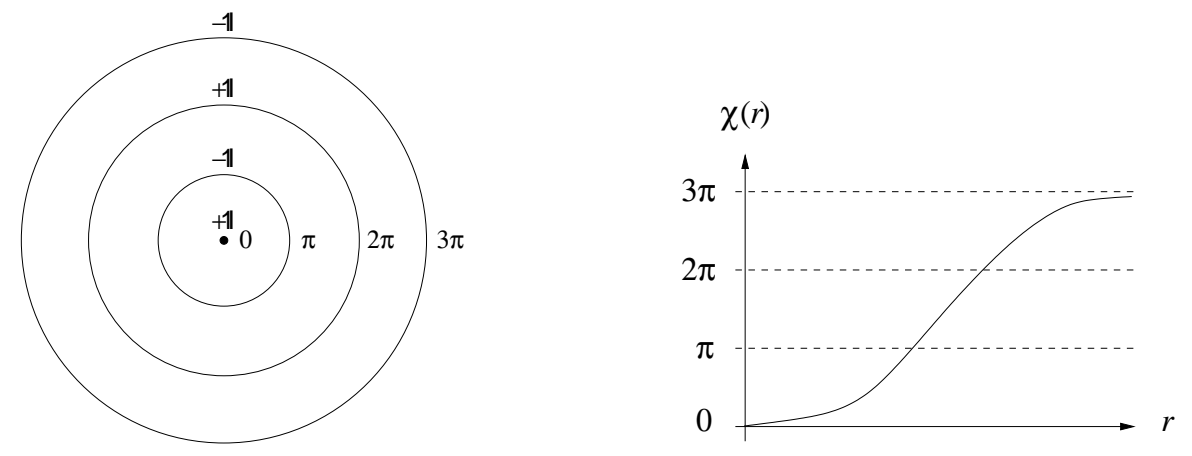

Figure 2: Smooth assignment of alcoves for a hedgehog type of mapping $\Omega: \dot{\mathbb{R}}^{3} \mapsto S U(2)$. (a) Defect structure of $\Omega$. (b) Profile $\chi(r)\left(\ln \omega=-i \chi \sigma_{3}\right)$ as a function of $r=|\mathbf{x}|$. The Weyl alcove is changed between the defects, such that $\chi(r)$ becomes globally smooth.

1. Choose the alcoves in every connected region such that $\tilde{\chi}(r)$ is globally smooth, i.e. $\tilde{\chi}(r)=$ $\chi(r)$, see fig. 2.

2. Exploit the arbitrariness $\chi \rightarrow \chi+2 \pi$ and the Weyl symmetry $\chi \rightarrow-\chi$ to fix the Weyl alcove $\tilde{\chi} \in[0, \pi]$ for all connected regions of $\dot{\mathbb{R}}_{\epsilon}^{3}$, i.e. $\tilde{\chi}(r)$ is the profile obtained from the original $\chi(r)$ by reflections at the boundary of the alcove, see fig. 3. While both $\omega(\mathbf{x})$ and $\ln \omega(\mathbf{x})$ are still continuous, they fail to be smooth across the domain walls.

Note that we may enclose both the monopoles and the domain walls by simple 2-spheres $S^{2}$ so that it is convenient to switch to spherical coordinates $\mathbf{x} \rightarrow(r, \vartheta, \varphi)$ on $\dot{\mathbb{R}}^{3}$. We may cover every connected region in the punctured space $\dot{\mathbb{R}}_{\epsilon}^{3}$ by just two coordinate patches $\mathbf{X}_{ \pm}$, so that their intersections with the wrapping sphere $S^{2}$ yield the northern $\left(S_{+}^{2}\right)$ and southern $\left(S_{-}^{2}\right)$ hemisphere. In each of these contractible patches, we find a smooth diagonalising coset lift $V_{ \pm}(r, \vartheta, \varphi)$.

In the case of a smooth choice of $\chi(r)$ (see fig. 2), the hedgehog $\Omega$ can be diagonalized by the same set of coset lifts $V_{ \pm}$for all $r$. An explicit form is given by [5],

$$
V_{+}(\mathbf{x})=\exp \left(i \frac{\vartheta}{2} \mathbf{e}_{\varphi} \boldsymbol{\sigma}\right) ; \quad V_{-}(\mathbf{x})=h_{ \pm}(\mathbf{x}) \cdot V_{+}(\mathbf{x})
$$

where the transition function

$$
h_{ \pm}=\exp \left(i \varphi \sigma_{3}\right)
$$

has support in the overlap between the two hemispheres, i.e. on the equator $S^{1}$ defined by $\vartheta=\frac{\pi}{2}$. From these expressions, we can easily calculate the magnetic flux of the induced Abelian potential $\mathcal{A}_{ \pm}^{3}=\left(V_{ \pm} d V_{ \pm}^{-1}\right)^{3}$. The magnetic field is always directed radially outwards (pointing to infinity), and to every wrapping surface, we assign a magnetic charge (48)

$$
m=-\frac{1}{4 \pi} \sum_{ \pm} \int_{S_{ \pm}^{2}} d \mathcal{A}_{ \pm}^{3}=-\frac{1}{4 \pi} \int_{S_{+}^{2} \cap S_{-}^{2}}\left(\mathcal{A}_{+}^{3}-\mathcal{A}_{-}^{3}\right)=\frac{1}{2 \pi} \int_{\text {equator } S^{1}} d \varphi= \pm 1 .
$$

Recall that the sign of this charge is determined by the orientation of the intersection $\mathrm{X}_{\alpha} \cap \mathrm{D}_{i}^{\epsilon}$, which is opposite to the orientation of the wrapping surface. Thus, if the surface $\partial \mathrm{D}_{i}^{\epsilon}$ encloses the defect from the outside (i.e. it is closer to infinity than the defect), the intersection $\mathrm{X}_{\alpha} \cap \mathrm{D}_{i}^{\epsilon}$ 

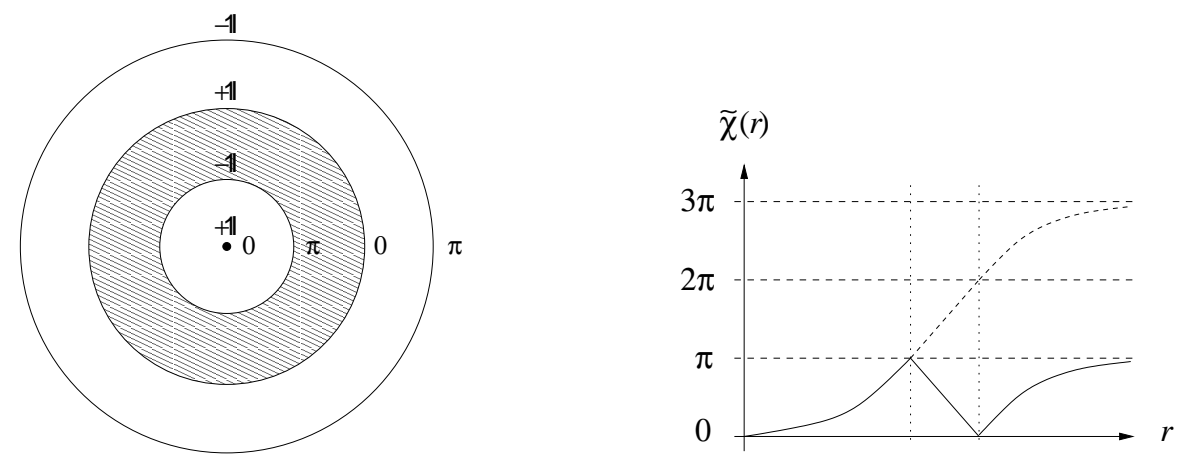

Figure 3: Fixed assignment of alcoves for a hedgehog type of mapping $\Omega: \dot{\mathbb{R}}^{3} \mapsto S U(2)$. (a) Defect structure of $\Omega$. (b) Profile $\tilde{\chi}(r)\left(\ln \omega=-i \tilde{\chi} \sigma_{3}\right)$ as a function of $r=|\mathbf{x}|$. The Weyl alcove is rigidly fixed to $\tilde{\chi} \in[0, \pi]$ such that $\tilde{\chi}(r)$ is reflected at the alcove boundary, i.e. the profile is continuous, but not smooth.

is aligned with the magnetic field whence the defect has charge $(+1)$. On the other hand, a wrapping surface closer to the origin than the defect is given a charge (-1) (see also fig. (1).

Thus, for a smooth choice of profile $\chi(r)$ (see figs. 2 and 1 (left)), the magnetic charges of the two surfaces $S^{2}$ wrapping the domain wall cancel and there is no net (intrinsic) magnetic charge on the domain walls. Hence the magnetic field goes smoothly through the domain wall without noticing its existence. Thus for a smooth choice of $\chi(r)$ the domain walls do not contribute to the Pontryagin index. The Pontryagin index is entirely determined by the two monopoles at $r=0$ and $r=\infty$. In fact since $\chi(0)=0$, only the monopole at infinity contributes. From $\chi(\infty)=n[\Omega] \pi$ and the magnetic charge $m_{\infty}=-1$ of this monopole, eq. (50) yields the correct winding number.

Consider now the alternative case of a fixed assignment of alcoves as in fig. 3. In order to reflect $\tilde{\chi}(r)$ in the alcove $[0, \pi]$, we need to combine the coset lift $V_{ \pm}$from the smooth case above with a Weyl flip in the transition functions $h_{ \pm}$. This in turn leads to a change in the orientation of the magnetic field inside the shaded region of fig. 3: In this domain, the field is directed radially inwards, i.e. towards the origin, while we have the usual (outward) orientation in the remaining space. The orientation of the wrapping surfaces is still determined by a normal vector pointing towards the defect. Taking the specific example $n=3$ for simplicity (see figure 4 (right)), we encounter the following defects:

- The monopole in the origin is a $(+\mathbb{1})$-defect and does not contribute to $n[\Omega]$.

- The first domain wall has two wrapping surfaces. The inner one is directed outwards (towards the domain wall) and this coincides with the orientation of the magnetic field. From the rules explained above, it is assigned a charge $m^{-}=-1$. The outer wrapping surface is directed inwards (towards the defect) and this also coincides with the flipped orientation of the magnetic field in the shaded region. It thus also carries a charge $m^{+}=$ -1 . Altogether, the first domain wall hence carries a total magnetic charge $m=-2$.

- The next domain wall is a $(+\mathbb{1})$-defect and again does not contribute. However, it carries magnetic charge +2 and flips the magnetic field back to pointing outwards.

- The monopole at infinity is a $\Omega=-\mathbb{1}$ defect and has charge $(-1)$ since the magnetic field has again its standard orientation pointing to infinity. 

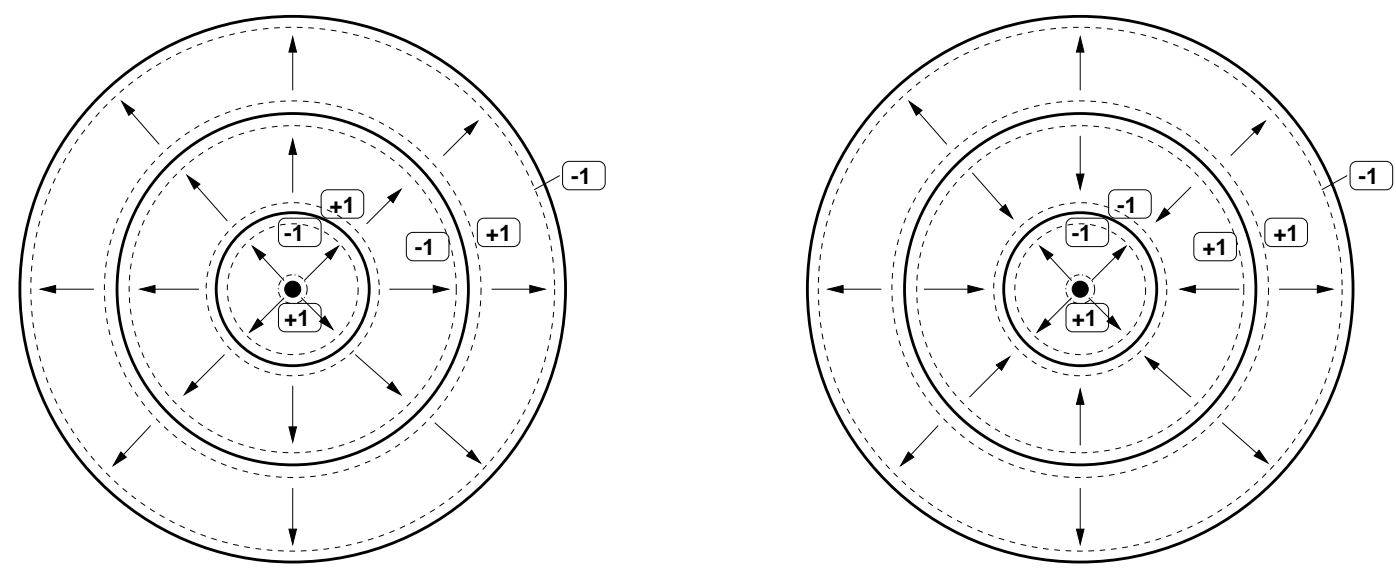

Figure 4: The $n=3$ hedgehog mapping with continuous profile (left) and restricted profile (right). Solid circles represent domain wall defects, dashed circles represent the wrapping surfaces and the dot in the origin symbolises the magnetic monopole. The numbers in the boxes denote the magnetic charge of the respective wrapping, which is determined from the relative orientation of the surface (not indicated for clearity) and the magnetic field (the radial arrows).

With these observations, our formula (52) gives the correct result

$$
n[\Omega]=-[(-2)+(-1)]=+3 .
$$

It should be noted that the similar analysis of refs. [6, 7], did not take domains walls into account, and thus erroneously predicts $n[\Omega]=1$ for the presently considered example.

\section{Summary and Conclusions}

We have investigated the topological charge of Yang-Mills fields in Polyakov gauge. Our main results are given by eqs. (50) and (51) and can be summarized as follows: If the temporal gauge field $A_{0}(x)$ is chosen smoothly as in [5], the Pontryagin index of a generic field configuration is entirely given by magnetic monopoles. On the other hand, if $\chi=\beta A_{0}$ is restricted to the first Weyl alcove, in addition magnetic charges for the closed domain walls arise which also contribute to the Pontryagin index, see eq. (52).

Other, open, magnetically charged defects, like open domain walls or lines, are topologically equivalent to magnetic monopoles and can be treated in the same way.

Although these results have been obtained in Polyakov gauge, we believe that they are generic for all Abelian gauges. In fact, recent lattice calculations performed in the maximum Abelian gauge [12] show also clear correlations between the (topological charge of the) instantons and magnetic monopoles.

\section{Acknowledgement:}

We thank M. Engelhardt for a careful reading of the manuscript and critical remarks.

\section{References}

[1] G.S. Bali, V. Bornyakov, M. Mueller-Preussker, K. Schilling, Phys. Rev. D54 (1996) 2863. 
[2] G. 't Hooft, Nucl. Phys. B190[FS3] (1981) 455.

[3] A. Di Giacomo, talk presented at QCD '98 (Montpellier) hep-lat/9809014.

[4] T. Banks, A. Casher, Nucl. Phys. B169 (1980) 103.

[5] H. Reinhardt, Nucl. Phys. B503 (1997) 505.

[6] C. Ford, U. G. Mitreuter, T. Tok, A. Wipf, J.M. Pawlowski, Monopoles, Polyakov-Loops and Gauge Fixing on the Torus, hep-th/9802191.

[7] O. Jahn, F. Lenz, Structure and Dynamics of Monopoles in Axial-Gauge QCD, hepth/9803177.

[8] R. Jackiw, Rev. Mod. Phys. 52/4 (1980) 661.

[9] K. Johnson, L. Lellouch, J. Polonyi, Nucl. Phys. B367 (1991) 675.

[10] H. Reinhardt, Mod. Phys. Lett. A11 (1996) 2451.

[11] M. Blau, G. Thompson, Comm. Math. Phys. 171 (1995) 639.

[12] H. Markum, W. Sakuler, S. Thurner, Nucl. Phys. Proc. Suppl. 47 (1996) 254.

M. Feuerstein, H. Markum, S. Thurner, talk presented at Hirschegg '97, hep-lat/9702006. 